\title{
PABLO PALAZUELO. ESPACIO PLEGADO
}

\author{
Gonzalo Sotelo Calvillo \\ Colaborador de la Fundación Pablo Palazuelo ${ }^{1}$
}

\begin{abstract}
Pablo Palazuelo (1915-2007) no sólo demostró su maestría en el ámbito pictórico, sino que también se adentró con éxito en la tercera dimensión. A partir del análisis de los fondos de la Fundación del artista se ha tratado de reconstruir el proceso de trabajo escultórico, empleando como semilla los trazados simbólicos contenidos en el boceto Del cuatro para seguir el rastro de las maquetas y croquis inéditos que custodia dicha institución.

Palabras clave: Pablo Palazuelo; proceso; dibujo; maqueta; escultura; Fundación Pablo Palazuelo; Pere Casanovas.
\end{abstract}

\section{PABLO PALAZUELO. FOLDING SPACE}

Pablo Palazuelo (1915-2007) not only demonstrated his command of the pictorial field, but also ventured successfully into the third dimension. Based on the analysis of the holdings of the Pablo Palazuelo Foundation, the author reconstructs the artist's sculptural process, using as a basis the symbolic lines found in the sketch Del cuatro, and tracing unpublished models and sketches in the collection of the Foundation.

Key words: Pablo Palazuelo; Process; Drawings; Model; Sculpture; Pablo Palazuelo Foundation; Pere Casanovas.

Como citar este artículo/Citation: Sotelo Calvillo, Gonzalo (2017): "Pablo Palazuelo. Espacio plegado". En: Archivo Español de Arte, vol. 90, núm. 357, Madrid, pp. 67-81; doi: 10.3989/aearte.2017.05.

La obra de Pablo Palazuelo (Madrid, 1915-Galapagar, 2007) representa un diario de su vida². Un recorrido que le llevó hasta la Universidad de Oxford para cursar estudios de arquitectura (1933-1936). Tras su regreso a Madrid redirigió la línea investigadora emprendida en dicha universidad hacia la pintura, donde emprendió una transición hacia la no-figuración en paralelo a una febril búsqueda de nuevas referencias teóricas que sustentasen sus composiciones. Una exploración que se vio acelerada con la concesión en 1948 de una beca del Gobierno Francés que le permitió emprender su segundo viaje iniciático, en esta ocasión rumbo a París.

Además de compartir estudio con Eduardo Chillida, Palazuelo residió en el Barrio Latino de París en cuyas librerías adquirió los libros que fueron nutriendo su biblioteca, donde se reunía un personal sincretismo conciliador entre los avances científicos y los saberes místicos. Apadrinado por la galería Maeght, no sólo desarrolló una importante producción pictórica, sino que también se aventuró a efectuar el salto a la tercera dimensión. Sin embargo, el comienzo de esta nueva senda no fue fácil. A la opinión crítica del director de Maeght, Louis Clayeux, al contemplar su primera obra tridimensional, Ascendente $(1954)^{3}$, se sumó la decepción de no ver materializadas a gran escala las maquetas realizadas para los salones Hisa (1967) de Madrid.

\footnotetext{
1 gonzalo.soteloc@gmail.com / ORCID iD: http://orcid.org/0000-0001-7811-8293

2 Biografía en Palazuelo/Esteban, 1980: 205-208.

3 Reproducido en Palazuelo/Esteban, 1980: 180.
} 


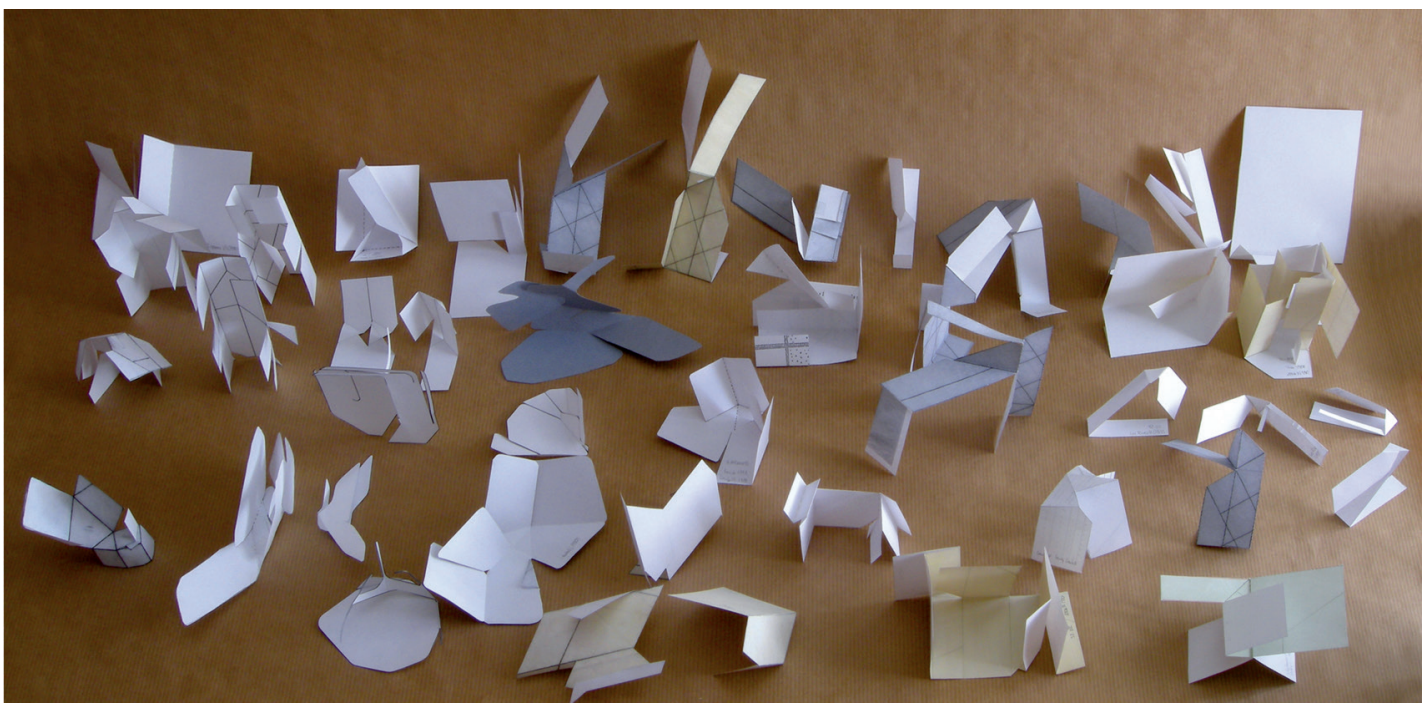

Fig. 1. Selección de maquetas realizadas en papel por el autor a partir de los diseños analizados.

Una vez establecido nuevamente en España, Palazuelo decidió participar plenamente en el gran desafío de la escultura. Aunque contaba con una edad avanzada -sesenta años-, su inmersión definitiva se produjo en 1975 al formar sociedad productiva con el escultor Pere Casanovas, afincado en el municipio barcelonés de Mataró. A lo largo de su trabajo como socios, el grado de implicación de Casanovas varió a partir de una primera fase en la que Palazuelo aportaba al taller diseños y maquetas de zinc indistintamente, con instrucciones muy precisas, fruto de una concepción solitaria y profundamente heredera del dibujo.

Durante esta época, la función del taller del Maresme consistía principalmente en realizar pruebas sobre materiales, al cambiar la escala y espesores alcanzados en las maquetas para trasladar las formas hasta las dimensiones de la escultura final, respetando las medidas y los ángulos prefijados. Posteriormente, el escultor catalán pasó a participar en un diálogo dentro del proceso de trabajo, un punto de inflexión que éste fijaba a partir de la producción de la obra Caixa II $(1980)^{4}$. A lo largo de este segundo período, se establecía un recorrido procesal que, -aunque no siempre incluía todos los pasos intermedios- podían llegar a transcurrir a través de bocetos iniciales, maqueta de idea, maqueta de taller, plantillas de corte, prueba de taller y pieza definitiva.

Se trataba de un trabajo en equipo, fundado en una dialéctica donde se adoptaban decisiones que hacían referencia a obras precedentes respecto a grosores, escalas, dimensiones, materiales y apoyos. En los últimos años de su vida, Palazuelo se limitaba a volver a transformar los pliegues y cortes de maquetas ya existentes, llevando los cambios hasta el extremo de destruir algunas de ellas. Debido a esta pérdida, no es posible rastrear todas las trazas que describieron muchos de los modelos finales durante su producción. Por este motivo se podría poner en valor el análisis desarrollado en la presente investigación, al permitir devolver a la materialidad mediante réplicas, muchas de las maquetas desaparecidas (fig. 1).

Al tener acceso a los bocetos de Palazuelo, se ha podido reconstruir parcialmente la senda transitada por estos diseños, lo que también permite fijar una consonancia en la manera de operar dentro de la evolución de las obras bidimensionales. Palazuelo explicaba el salto a la tercera dimensión como un impulso, una necesidad, para hacer más evidentes los poderes de transformación formal operados por el número y la geometría. De este modo trataba de mostrar las relaciones espaciales y volumétricas, ya que la forma deviene más accesible al dinamismo de las

\footnotetext{
${ }^{4}$ Comunicación con Casanovas. Mataró: 17 de marzo de 2007.
} 
energías cambiantes, generadoras de formas en constante modificación, y al movimiento inmóvil. Para representar la tensión y la materia en su obra gráfica empleaba el color -interacción de luz y materia-, y en la escultórica esta función de heraldo recaía en el espacio interior. Entendía el espacio como vacío activo, poblado de materia y energía, por lo que constituía una matriz de infinitas formas en potencia.

Reafirmándose en su huida del encorsetamiento este nuevo medio le permitió trascender la dictadura de los límites que fija el marco del lienzo, o las fronteras impuestas por el papel. La nueva configuración del espacio le permitía definir los propios confines de cada pieza; sus extremos seguían siendo precisos, pero esta vez podrían ser producto de una decisión activa del autor. Palazuelo defendía una concepción del vacío próxima a los planteamientos budistas, y coincidente también con las teorías de los científicos contemporáneos, al presentarlo repleto de energía, de vibración y, por tanto, de formas. Todas las lecturas que cultivaba se referían a la necesidad de abrirse a esta energía, materializada en un componente gráfico. Por este motivo fatigó tratados geométricos que traducían las teorías que cimentaban su pensamiento a través de los mandalas estudiados por Jung y Von Franz ${ }^{5}$, o los yantras investigados por Mookerjee ${ }^{6}$. Pero también los patrones de teselación de Penrose ${ }^{7}$ o los trazados reguladores de Le Corbusier ${ }^{8}$. Un principio que trató de trasladar a la configuración del espacio escultórico articulado a partir de sus dibujos.

El propio artista señalaba su producción tridimensional deudora del ámbito gráfico: "En mi trabajo los dibujos constituyen lo que se puede llamar el germen o la semilla de las obras que de ellas proceden. Las esculturas son el desarrollo en la tercera dimensión de mis formas bidimensionales" ". Salvo contadas excepciones, la deuda con su obra sobre papel es evidente al emplear planos en detrimento de un volumen que deja de ser matérico para configurar espacios activos. Así, Palazuelo sostenía en una entrevista mantenida con Félix Guisasola en 1981: "Me interesan sobre todo las superficies, por lo tanto la plancha de metal es el material que se me impone como mejor soporte para plasmar lo que pretendo" 10 . Además se reafirmaba en la necesidad del concurso de estas superficies para lograr configurar volúmenes abiertos: "no envuelvo el espacio hasta cerrarlo. Me atraen más las superficies que se abren y despliegan en el aire. Por el momento, me interesa menos la rotundidad, lo que tiene el volumen de terminado, de definitivo"11.

Este postulado, donde establecía que el origen de su escultura residía en el desarrollo de sus formas bidimensionales, las cuales constituirían la semilla estructuradora que generaría nuevas familias que saltaban hasta la tercera dimensión parecía categórico. Sin embargo, existía una correspondencia bidireccional entre escultura y pintura, dado que -como señalaba Casanovas-, Palazuelo llegó a afirmar "mediante la escultura evoluciona mi pintura"12. Ergo, no sólo existía una influencia desde la obra gráfica, sino que ésta fluía en ambos sentidos. Por este motivo, deseaba exponer pintura acompañada de escultura, dado que se compenetraban. "Las irradiaciones del punto son dimensión real, manifiesta por 'pulsación rítmica' (energía) que, al penetrar en el espacio, lo coagula en planos. Este pulsar rítmico es 'número'"13.

Durante la traducción de los trazados gráficos a la tercera dimensión, en el taller de Casanovas se eliminaron las evidencias del proceso, retirando del material una memoria que podría distraer la transmisión de su mensaje. Un método que concuerda con la planeidad que Gállego ${ }^{14}$

\footnotetext{
5 Franz [1970], 1974: 171-211.

${ }^{6}$ Khanna/Mookerjee, 1979.

7 Penrose, [1989] 1991: 210-216, 620-622.

${ }^{8}$ Le Corbusier, [1923] 1946: 76.

9 Palazuelo, 2004: 2.

${ }_{10}$ Palazuelo/Guisasola, 1981: 24.

11 Palazuelo/Guisasola, 1981: 24

12 Comunicación con Casanovas. Mataró: 26 de enero de 2013.

13 Palazuelo/Amón, 1976: 30.

14 Gállego, 1976: 53-58.
} 
encontraba en la superficie de sus lienzos, donde se hurtaba el gesto del pincel, por lo que se lograba una obra coherente de la que se omitían las huellas del combate que apuntaba Claude Esteban $^{15}$.

El proceso seguido transitaba por diversas fases hasta alcanzar el deseado resultado final. Durante su entrevista con Kevin Power, Palazuelo no sólo declinó ocultarlas, sino que procedió a describirlas minuciosamente:

Hago un plano que procede de uno de mis dibujos antiguos o modernos, de hace unos quince años. Este dibujo lo paso a una chapa de metal, generalmente zinc o aluminio fino para poder manipularlo. Entonces lo pliego según las líneas que marcan las formas que son como límites de planos, atendiendo a un orden que corresponde a ese plano. [...] Es decir, que la estructura plana se convierte en una estructura hija tridimensiona ${ }^{16}$.

Se ha procedido a desarrollar una investigación que arrancaba con el rastreo de las piezas terminadas a partir de las huellas conservadas de su proceso de producción. De esta manera, se ha tratado de establecer un registro temporal que aglutinase diseños pictóricos, bocetos e incluso maquetas. Se han redibujado las trazas presentes en los croquis con una jerarquía de línea que distingue las aristas de doblez y corte -discontinua y granate-. A su vez, se han realizado comprobaciones de las premisas adoptadas al elaborar maquetas en papel y cartón a partir de los diseños destilados.

En este artículo se reúne una porción significativa de las propuestas que ilustran la aproximación que obró Palazuelo durante sus investigaciones esculturales. A partir de una selección de los arquetipos más feraces empleados por el escultor, se propone un viaje que trata de desvelar las organizaciones geométricas implícitas en su generación estructural. La singularidad de este texto, sólo ha sido posible a partir del descubrimiento de las legiones de maquetas, bocetos y croquizaciones inéditos, que el pintor madrileño guardaba celosamente. A raíz del trabajo de catalogación efectuado sobre los fondos pertenecientes a la Fundación Pablo Palazuelo, se han podido desvelar muchas de las sinergias organizativas empleadas ${ }^{17}$.

Sin embargo, estos dibujos no se almacenaron siempre agrupados, por lo que es posible descubrir elementos invariantes que podrían servir de puentes entre las distintas series formales, trascendiendo las barreras temporales. Por lo que en la ordenación de dicho inventario se ha adoptado como unidad la carpeta, y obedece al deseo de respetar la disposición de los archivos encontrada originalmente en el taller de Palazuelo. Dentro de la descripción de las distintas obras se incluye un código ideado para la catalogación en la Fundación Pablo Palazuelo, que comienza con las iniciales de la institución, seguidas del número de carpeta y número de obra separados por un guión: FPP XX-XXX.

Sin duda, el mayor potencial para el paso de su obra a la tercera dimensión residía en diversos diseños elaborados tanto en París como en Monroy. Partiendo de esquemas muy sencillos, la imparable evolución de las líneas soñadoras convirtieron los trazos de Palazuelo en aristas de pliegue o corte, hasta coagular la obra gráfica en planos metálicos. Definían un volumen vacío confinado por la emergencia de superficies que lo envolvían en una suerte de ejercicio de papiroflexia. El proceso parecía obedecer a las premisas del orden implicado enunciado por el físico David Bohm ${ }^{18}$, donde dicha ordenación holográfica se materializara en la escultura y se explica desplegado en las obras bidimensionales. También encontraría similitud con los movimientos de estirado y plegamiento sucesivos, descritos por Stewart ${ }^{19}$, que funcionan como herramientas es-

\footnotetext{
15 Palazuelo/Esteban, 1980: 179.

16 Palazuelo/Power, 1995: 76.

17 Por lo que quisiera agradecer la generosa colaboración de dicha institución para la elaboración de este escrito, y el testimonio brindado por el escultor Pere Casanovas

18 Bohm, 1980: 240-248.

19 Ian Stewart explicaba que Stephen Smale empleó este movimiento, conocido como la "transformación del panadero", para lograr una estructura de gran complejidad. Stewart, 1991: 154.
} 
pecialmente eficaces en la consecución de estructuras no lineales, dentro del estudio de sistemas caóticos.

Por medio de este proceso de plegado de sucesivos planos se obtiene una unidad topológica formal en sus esculturas. Las trazas de pliegue y corte aplicadas sobre las planchas metálicas de los modelos generan un equilibrio dinámico. La importancia del plegado en esta concepción aparece subrayada en el ejemplar de la biblioteca de Palazuelo del libro El pliegue. Leibniz y el Barroco (1988), donde Deleuze aporta una componente material al afirmar: "La unidad de materia, el más pequeño elemento del laberinto es el pliegue, no el punto, que nunca es una parte, sino una simple extremidad de la línea. Por eso las partes de la materia son masas agregadas, como correlato de la fuerza elástica compresiva" ${ }^{20}$. Conformaba soluciones metálicas envueltas que parecen retomar los experimentos espaciales acometidos en los talleres de los Vkhutemas ${ }^{21}$ rusos en los años veinte del siglo XX, que seguían la estela constructivista del Manifiesto Realista (1920) de sus admirados Gabo y Pevsner ${ }^{22}$.

Debido a los múltiples mecanismos transformadores operados desde la fase gráfica para alcanzar el estadio final, se ha estimado que desplegar las piezas escultóricas podría constituir una manera operativa para desvelar dichas correspondencias hasta obtener su orden explicado. De esta manera, aflorarían las posibles filiaciones subyacentes presentes en las distintas obras, sin someterse exclusivamente a las relaciones nominales o cronológicas.

\section{Variaciones Del cuatro}

De todas las simbologías empleadas por Palazuelo, Del cuatro es la que desencadenó una mayor variedad de soluciones, poniendo en juego tan sólo dos piezas. Resulta difícil fijar el origen de estos arquetipos, dado que los bocetos desvelados en las carpetas del pintor que los contienen no aparecen datados. La primera aparición que alumbraba cierta seguridad cronológica surgió durante la croquización del proyecto para el techo de la residencia Huarte en Madrid, por este motivo la fecha más antigua se remontaría hasta 1965. Se tratan de dos sencillas figuras rectangulares que, a su vez, están subdivididas por una serie de diagonales, verticales y horizontales que se despliegan en su interior hasta generar diversos trapecios. Comparten una geometría que podría recordar tanto a los paisajes abstractos trazados por Paul Klee ${ }^{23}$-cuyas obra fue estudiada desde el ámbito geométrico por Bouleau ${ }^{24}$-, como a las descomposiciones armónicas del rectángulo que estudió el matemático Matila Ghyka ${ }^{25}$.

La combinación de ambos moldes ancestrales y la extracción selectiva de algunos de sus trazados habilita una diversidad prácticamente infinita de variaciones. Estas composiciones se ayudaban de las posibilidades que brinda la transparencia del papel vegetal a la hora de incluir la variable de simetría entre las operaciones que rigen traslaciones y giros. A modo de muestrario, una serie de posiciones se ilustraba en la hoja catalogada en la Fundación como FPP 16-064, publicada por primera vez en la monografía que le concedió Maeght en $1980^{26}$. Con grosores jerarquizados se pueden apreciar combinaciones de cuatro, seis u ocho piezas con un sistema de ensamblaje que siempre dicta la necesidad de unir las unidades según una disposición longitudi-

\footnotetext{
20 Deleuze, [1988] 1989: 14.

${ }^{21}$ Ivanova-Veen/Ovsiannikova, 2005: 97.

22 Tanto en este manifiesto como en Escultura: tallar y construir en el espacio (1937), los hermanos Gabo y Pevsner mostraban su desaprobación por el arte contemporáneo. Proponían desplazar el interés desde la talla hacia la construcción, desde la masa hasta el espacio, para proponer una visión estereométrica que se aproxima al protagonismo plano de las superficies plegadas por Palazuelo. Chipp, [1968] 1995: 350-363.

${ }_{23}$ Composiciones de una linealidad no figurativa como la obra de 1934 Bergdorf (herbstlich) [Pueblo de montaña (otoñal)]. Óleo sobre lienzo, 71,5 × 54,4 cm. Guigon/Llorens, 1998: 197.

24 Bouleau, [1963] 1988: 291.

25 Ghyka, [1946] 1977: 128-134.

26 Palazuelo/Esteban, 1980: 182.
} 


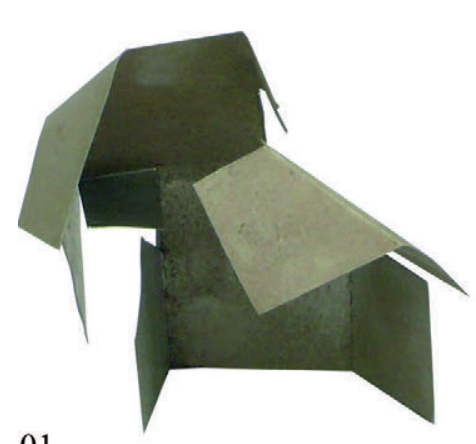

01 .

Fig. 2: 01. Palazuelo, Pablo, c. 1977. Maqueta $N .^{\circ} 12$ [Del cuatro I]. Chapa de aluminio. FPP. 02. Palazuelo, Pablo, c. 1985. Maqueta s.t. [Arquitectura IB]. Chapa de zinc. FPP. 03. Palazuelo, Pablo, s.f. Maqueta N. ${ }^{\circ} 12$. Chapa de zinc. FPP. 04. Palazuelo, Pablo, s.f. Maqueta s.t. [Clinamen II]. Chapa de aluminio. FPP.

nal. Siguiendo estas sencillas reglas, Palazuelo desplegó no sólo sus posibilidades gráficas en los dibujos y gouaches Trazas del cuatro (1986-1987) sino que también las implementó dentro del ámbito escultural, logrado impactantes resultados.

Cuando se ha abordado el análisis de las piezas objeto de estudio se ha continuado efectuando un proceso de filiación referido a sus estructuras geométricas, alejado del facilismo de agrupaciones que obedezcan a los títulos o a los años de ejecución. Por consiguiente, los linajes se establecen en función de las diversas permutaciones efectuadas con los arquetipos los cuales se reconstruyen con tinta gris en los casos donde su simplificación dificulta su reconocimiento.

Las esculturas resultantes de este período formal se alejan de la masividad para envolver el espacio mediante una sucesión de esbeltos pliegues metálicos (fig. 2). Por tanto, Palazuelo conforma obras que se asemejan más a piezas como Locmariaquer IX (1989) ${ }^{27}$ de Chillida, o a las construcciones vacías de Jorge Oteiza, como Arista vacía $(1958)^{28}$. En un recorrido que arranca con el depurado dibujo $N .^{\circ} 12$ (1977, FPP 25-002), donde se jerarquizan las líneas para indicar distintos tratamientos del metal. Su enigmático y errático trazado cobra sentido al restituir las trazas del boceto original, para desvelar una estructura subyacente compuesta por la agrupación de cuatro figuras. Las grafías seleccionadas se convirtieron en aristas de pliegue y corte en la maqueta (fig. 2.01) que originó en 1977 la escultura Del cuatro I.

Tras una recolocación de los arquetipos geométricos, se destilaron los trazados para diversas esculturas a partir del diseño Del cuatro 3 (c. 1978, FPP 41-024). En 1978 se desarrollaron nuevos experimentos con la escala y el material, al representar el ámbito de tránsito por medio de la formalización de Les Portes I (1978) ${ }^{29}$. Realizada con tres planchas de acero inoxidable de un metro de lado, los cortes y dobleces son minimizados en favor de la reproducción de los trazados lineales mediante una suerte de bajorrelieve.

Con ligeras variaciones, estas mismas trazas son plasmadas de nuevo en Del cuatro II (1978, FPP 25-006), regulador de piezas elaboradas en cobre pulido y diferenciadas únicamente por su escala. Nombradas Del cuatro II y III $(1978)^{30}$, estas esculturas obedecen a una misma geometría, pero no la evidencian tan claramente como en Les Portes I (1978) al desaparecer las marcas que surcan las superficies metálicas. Esta serie no acabó en estas dos obras, sino que su colofón

27 Escultura realizada en acero cortén, $100 \times 100 \times 100 \mathrm{~cm}$. Barañano, 1998: 178 .

28 Escultura realizada con una composición a partir de unidades Malévich en acero, $95 \times 35 \times 38 \mathrm{~cm}$. Rowell/ Badiola, 2005: 260.

${ }^{29}$ Palazuelo/Esteban, 1980: 195.

30 Palazuelo/Esteban, 1980: 201. 
aconteció seis años después con la producción de la pieza Del cuatro $I V(1984)^{31}$ que representa una inversión de las trazas mostradas en el boceto Del cuatro IV (1983, FPP 25-003), donde incluso se especifica la altura de la escultura. Independientemente de sus diferencias concretas, en las obras de esta serie habita un patrón común que contiene un conjunto de pliegues verticales que acotan el espacio interior, además de dotar a la escultura de estabilidad.

Un esquema que se perpetúa en una producción tridimensional, aunque varíe su trazado regulador. En este modelo se tomó como referencia el dibujo Del cuatro 4 (1983, FPP 20-156) que muestra una agrupación de seis arquetipos, ya presente en el citado boceto reproducido en la monografía de Maeght en 1980. Cada conjunto de dos piezas provoca una arista de pliegue vertical que articula la maqueta metálica (fig. 2.02), sobre cuyo envés aún se conservan las líneas de grafito que reproducen su estructura subyacente. Desde estos planteamientos se construyó en 1985 la escultura Arquitectura IB.

Tras experimentar con la permutación de seis piezas, el escultor madrileño recondujo su investigación a los conjuntos de cuatro, que fueron evolucionando hacia modelos que contenían un decreciente número de planos. El lacónico proceso que conlleva la reducción de planos y trazas originada en la combinación de los cuatro arquetipos, ligados con Del cuatro I (1977) se retomó con la reordenación exhaustiva llevada a cabo por Palazuelo entre noviembre de 1991 y marzo del año siguiente. En estos bocetos se reproducían las pautas generales y, en algunas ocasiones, incluso se jerarquizaron sus líneas para distinguir las aristas de corte con lápiz rojo e indicando mediante flechas las concavidades del plegado, además de dotarles de una catalogación numérica. Al aparecer numerados, los trazados aportan pistas para poder relacionarlos no sólo con ciertas maquetas, sino también con diversas esculturas.

Producto de una nueva operación de simetría y recorte de la base constituida por $N .^{\circ} 14$ (1992, FPP 46-105) brotó un molde formal que amalgamaba numerosos diseños. Así, el título del dibujo $N .^{\circ} 1,5,7,10,11,12$ (1991, FPP 46-104) indicaba la potencialidad que habitaba en su seno. En la maqueta para Levante II (2000) también resuenan ecos de la coronación de Aku II (1999); motivo que refuerza la decisión de abordar una catalogación ligada al proceso generador sin atender exclusivamente a cronologías o títulos. Este modelo marcado con el número 12 (fig. 2.03), se perfila mediante el plegado de una sucesión rectangular que envuelve un prisma abierto elevado sobre el suelo mediante un diedro vertical.

Si en este trazado se implementan sendas simetrías parciales se logran las líneas de doblez y sección que producen las diversas maquetas (fig. 2.04), preámbulo de la escultura Clinamen II (1988), como puede rastrearse en el análisis gráfico efectuado. Realizados con distintos grosores, los modelos tridimensionales barajaban por una parte las diferentes variables de color, brillo y reflejos relativos al acabado metálico, y por otra los distintos ángulos de pliegue que articulaban las relaciones geométricas entre sus planos. Una vez fijados estos parámetros con solvencia, sus coordenadas se tradujeron en la solución definitiva mate elaborada en acero oxidado.

Al acometer una simplificación mayor de las estructuras manejadas con anterioridad, la Plantilla $N^{\circ} 5$ (1992, FPP 46-103) sirvió como catalizador de sendas maquetas donde se exploraron las posibilidades que ofrecía una configuración en forma de $X$ (fig. 3). En el modelo marcado con el número 19 se optaba por una base horizontal que conectaba las palas diagonales inferiores, mientras que la señalada con los números 1 y 3 en distintos planos indicaba la ambivalencia posicional de la pieza. Una equipotencialidad que se ramificó en dos subespecies; con una vocación tendida próxima al mobiliario surgió Conjunción II (1990, FPP 32-015), expuesta en la muestra Pablo Palazuelo inédito. La colección del artista (2012) ${ }^{32}$, donde dos piezas agudas enfrentadas se unen por un plano horizontal, interrumpido por un pliegue diagonal (fig. 3.01). Cuatro años más tarde se construyó una escultura que desplegaba su efervescencia vertical al alzarse mediante la modificación del orden de plegado hasta obtener Solar I (1994).

\footnotetext{
31 Palazuelo, 1985: 15.

32 Sotelo/Oropesa, 2012: 49.
} 


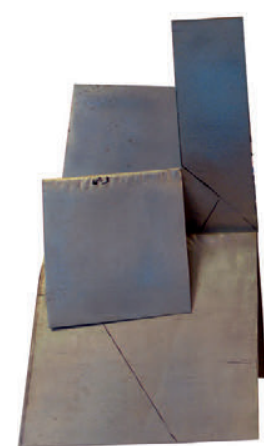

01

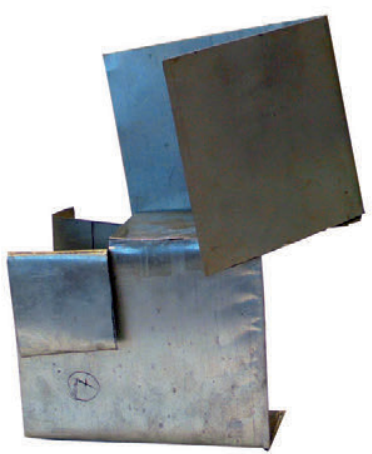

02.

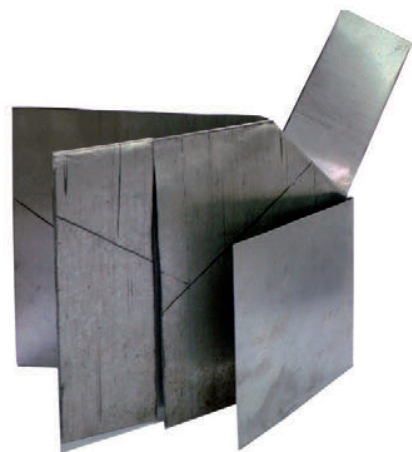

03.

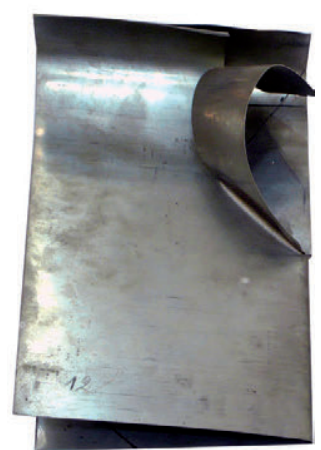

04.

Fig. 3: 01. Palazuelo, Pablo, s.f. Maqueta N. ${ }^{\circ} 16$ [Conjunción II]. Chapa de zinc. FPP. 02. Palazuelo, Pablo, 1995. Maqueta.$^{\circ}$ 7. Chapa de zinc. FPP. 03. Palazuelo, Pablo, s.f. Maqueta $N .^{\circ}$ 6. Chapa de zinc. FPP. 04. Palazuelo, Pablo, s.f. Maqueta N. ${ }^{\circ}$ 12. Chapa de zinc. FPP.

A partir de algunos diseños se obtuvieron obras que no siempre lograron trascender los límites del papel, aunque en ocasiones alcanzaron la fase de maqueta. Como ilustra el caso del modelo numerado con un siete (fig. 3.02), que articulaba la base geométrica contenida en la Plantilla $N^{\circ} 7$ (1991, FPP 46-102) para componer un espacio confinado por planos rectangulares. O proyectos que tuvieron que ser reconducidos como el montaje para la Galería Soledad Lorenzo (1996-97), que generó maquetas previas como la marcada con un seis (fig. 3.03) y heredera de la Plantilla N. ${ }^{\circ} 6$ (1991, FPP 46-110).

Durante los últimos años, Palazuelo volvió su mirada hacia maquetas antiguas, las cuales transformaba incluyendo el curvado de algunos de sus planos, quizás tratando de lograr una mayor afinidad con las familias orbitales que gestaba en este período. Sin embargo, fueron proyectos desechados que no llegaron a culminar en obras definitivas, ya que perdieron la fuerza de la idea inicial. De esta época, se conservan en los almacenes de la Fundación una maqueta de zinc, cuyo código numérico ha permitido establecer su filiación gráfica. Desde una simetría aplicada al diseño FPP 46-104, se obtiene un dibujo continente de nuevas plantillas, las $N{ }^{\circ}{ }^{\circ}$, $6,8,9$ (1991, FPP 46-117), que fue tanteada por una maqueta modificada a la que se rizaron los extremos para desarrollar el modelo N. ${ }^{\circ} 12$ (fig. 3.04).

Una vez agotadas estas transformaciones, incluso se retomaron antiguos diseños para continuar desenvolviendo sus posibilidades latentes. Si se analiza el boceto Para Atrio (c. 1986, FPP 20-157), se pueden reconocer las mismas pautas presentes en el dibujo $N .^{\circ} 12$ (1977, FPP 25002), a las que se han añadido verticales y horizontales equidistantes a líneas básicas que subdividen los distintos planos. Para establecer esta correspondencia entre ambos diseños en el redibujado de la investigación formal se han incluido en gris las trazas ausentes.

Tras seleccionar las líneas necesarias para configurar un espacio cuyo título evoca un atrio, se acometió una corroboración tridimensional (fig. 4). Representaba la voluntad de configurar un triedro vertical al que se suman sucesivos planos, coronados por una última doblez en forma de Y (fig. 4.01), presentes en la escultura Atria I (1986) ${ }^{33}$. Sus modificaciones no se agotaron en esta fase, dado que Casanovas comentaba la decisión de aplicarle una nueva transformación que le dotase de una mayor envergadura. Por esta razón, la pieza original se desmontó y se rehizo añadiéndole nuevas planchas metálicas hasta alcanzar los $230 \mathrm{~cm}$, renombrada como Difronte I (fig. 4.02). Las nuevas uniones se forjaron mediante cordones de soldadura que no se escamotearon en esta ocasión para evidenciar sus cicatrices, testigos de la metamorfosis sufrida, acompañando a los trazados en bajorrelieve que surcan geométricamente sus superficies. Se trata por

\footnotetext{
33 Reproducida en Arangüena, 1987: 20.
} 


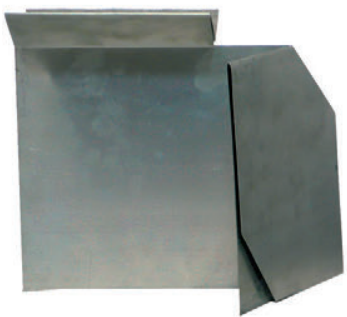

01 .

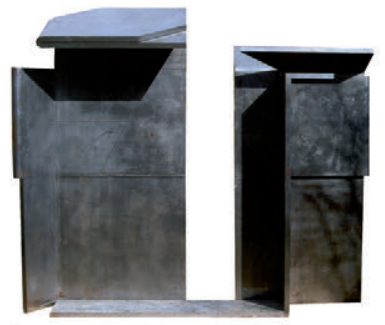

02 .

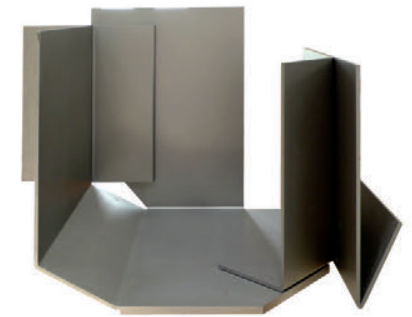

03.

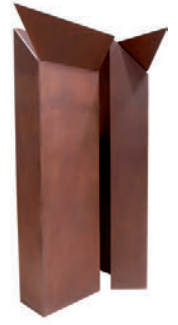

04.

Fig. 4: 01. Palazuelo, Pablo, c. 1986. Maqueta s.t. [Atria I]. Chapa de zinc. FPP. 02. Palazuelo, Pablo, 1986. Difronte I. Acero pulido, $230 \times 270 \times 85 \mathrm{~cm}$. FPP 32-048. 03. Palazuelo, Pablo, 1990. Atria III. Acero inoxidable fregado, $76 \times 71 \times 60 \mathrm{~cm}$. FPP 32-026.04. Palazuelo, Pablo, s.f. Umbral. Acero cortén, $250 \times 139 \times 107 \mathrm{~cm}$. FPP 32-004.

tanto de una obra singular en la que conscientemente no se omitieron las huellas que explican la memoria del material.

De vuelta al trazado original, Palazuelo siguió explorando la potencialidad que éste aún le brindaba para conformar un volumen dinámico confinado por planos replegados en diversas disposiciones, donde la superficie horizontal fue ganando protagonismo. Como ilustran las transformaciones operadas durante los cuatro años que separan Atria II (1986) de Atria III (fig. 4.03) hasta configurar distintas fortificaciones erigidas con planchas verticales que defienden un espacio interior mediante trazas poligonales abiertas.

Gracias a esta misma estructura geométrica, el escultor madrileño también pudo continuar sus investigaciones, desarrolladas en las esculturas Del cuatro (1977-84) y Arquitectura IB (1985), con distintos triedros de marcada verticalidad. Tras operar unas sencillas operaciones de copia y simetría, se obtiene la estructura geométrica que determinó la pieza Umbral I (1983), cuya prueba en oxi-cortén de dimensiones modestas fue escalada hasta los $250 \mathrm{~cm}$ (fig. 4.04). Conformaba unos estilizados muros, fatigados por grietas sutiles practicadas en sus superficies.

Si a la agrupación de cuatro arquetipos contenidos en Para Atrio (c. 1986, FPP 20-157), se le opera una simetría parcial sobre dos de ellos, se obtiene el trazado regulador de una nueva familia, que comenzó con Ikon I (1987) ${ }^{34}$. Escultura elaborada en hierro, en la que se mantiene la disposición vertical de los anteriores Umbral (1983), con la salvedad de que los pequeños cortes que se practican en el tercio inferior consiguen estabilizar una pieza extremadamente plana. Así mismo, una composición trapezoidal aflora por primera vez en su centro, sugerida con una lacónica ceja apenas perceptible en la superficie metálica (fig. 5).

Dos años más tarde, la sociedad formada por Palazuelo y Casanovas volvió a experimentar con el hierro, reforzando sus pies de sujeción. La escultura resultante de 1989, Ikon II (fig. 5.01), muestra la evolución de la figura trapezoidal desde su antecesora. Dentro de esta solución aparece taladrada y remarcada por una chapa que recorre su perímetro. Años más tarde y con esta misma forma se realizó la pieza Ikono III (1994).

La última variación derivada directamente de las trazas de la serie Ikon (1987-1994) se presentó en una disposición horizontal que volvía a incluir la oquedad trapezoidal hasta formalizar una topografía próxima al sentido arquitectónico de su título, Plaza (1998) ${ }^{35}$. En la maqueta preliminar (fig. 5.02) perviven nítidamente las trazas de lápiz que navegan sobre la lámina de zinc, además de un diedro que se erige orgulloso sobre el resto de la composición. Este proceso se resolvió en una escultura donde las huellas de grafito se tradujeron en sutiles hendiduras que desvelaban su origen geométrico, como anteriormente se había producido en Les Portes I (1978) o Difronte (1986), y se añadieron tres pequeños apoyos para elevar la plataforma horizontal.

\footnotetext{
34 Arangüena, 1987: 19.

35 Reproducida en Palazuelo, 1999: 67
} 


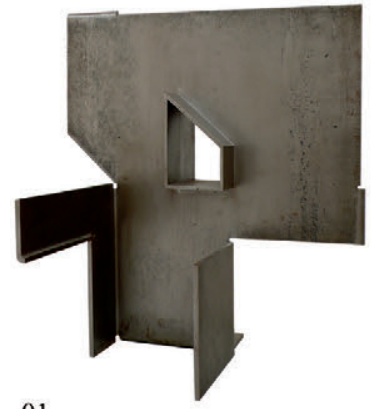

01.

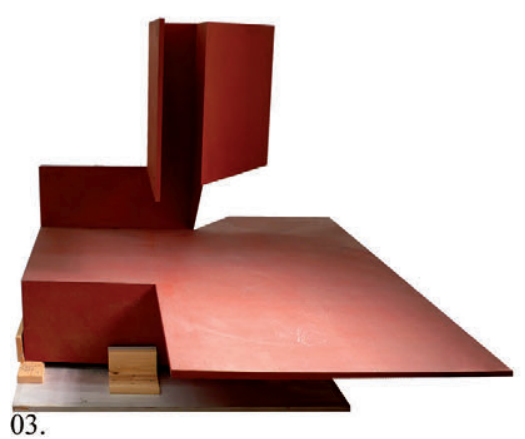

02 .
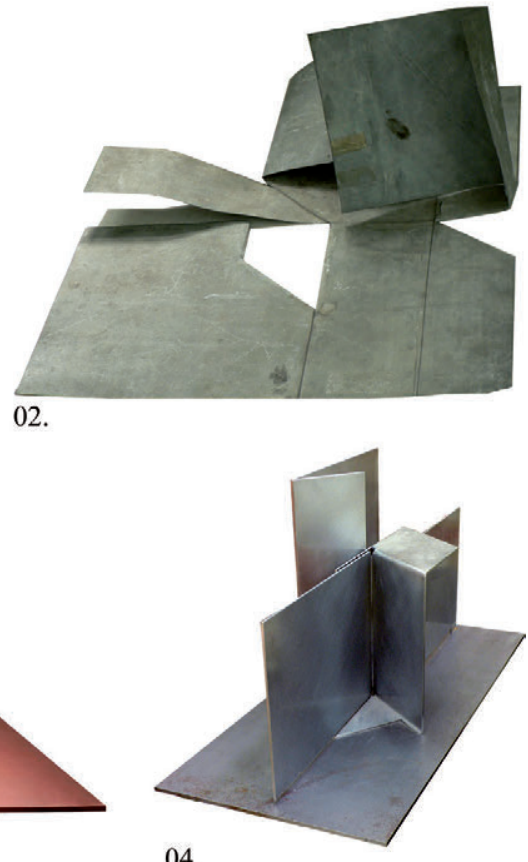

04.

Fig. 5: 01. Palazuelo, Pablo, 1989. Ikon II. Hierro, $60 \times 53,5 \times 41 \mathrm{~cm}$. FPP 32-019. 02. Palazuelo, Pablo, c. 1998. Maqueta s.t. [Plaza]. Chapa de zinc. FPP. 03. Palazuelo, Pablo, 1991. Plataforma II. Acero pintado, $115 \times 160 \times 145 \mathrm{~cm}$. FPP 32-031.04. Palazuelo, Pablo, 1998. Muro. Aluminio fregado, 40,5 ×83 × $24,5 \mathrm{~cm}$. FPP 32-028.

Sobre el hueco trapezoidal antes citado se situó la misma traza recortada, aunque soldada tras un giro de $180^{\circ}$ que desmiente su posible génesis a partir del pliegue.

Si en Ikon II (1989), Ikono III (1994) y Plaza (1998) se comenzaron a extender los planos más allá de los límites del boceto, posteriormente estas fronteras se tornaron más difusas. Así, para configurar la base de Plataforma II (fig. 5.03), se incluyeron nuevas chapas que cegasen las superficies verticales de la base de la escultura hasta elevarla sobre una suerte de podio. En su coronación también se planteó la presencia de un plano horizontal que cubriese los encuentros de remate, que finalmente fue retirado.

Unos planos más extensos se desplegaron para conformar la serena belleza de Plataforma I (1990), los cuales presentan un repliegue más intenso que refuerza la confrontación entre las superficies verticales y horizontales. Estas dos últimas esculturas comparten un aspecto singular, al tratarse de las dos únicas que escondían hasta esa fecha el acabado metálico bajo una capa de pintura, rojiza para la primera y negra para la segunda.

Por el contrario, si no se ensancha el ámbito del trazado sino que éste se acota seleccionando algunos de sus planos, también se alcanzaron notables obras, donde la pericia en los plegados suplió el menor número de elementos. Mediante una radical talla, la estructura geométrica se resumió en un conjunto seriado de rectángulos, modificado por dos diagonales que formalizan un rombo. Diferenciadas por una sucesión de dobleces y cortes, las diversas superficies se repliegan hasta configurar una especie de cerramiento sustentado mediante contrafuertes que aluden a su título constructivo: Muro (fig. 5.04).

Dentro de la croquización $N .^{\circ} 14$ (1992, FPP 46-105) se distinguen las mismas trazas que sirvieron para producir las primeras obras escultóricas de 1977, estudiadas al comienzo del presente artículo. A su vez, al seleccionar partes de la misma, también se obtienen nuevas estructuras geométricas (fig. 6). Así, al prolongar una de las verticales y omitir lo dibujado a partir de una nueva horizontal, brotaron los dibujos nombrados como Plantilla $N .^{\circ} 15$ (1992, FPP 46-115) 


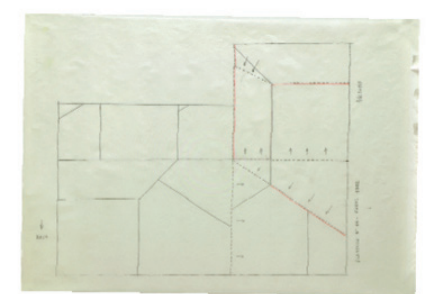

Plantilla No 14 (1992, FPP 46-116)

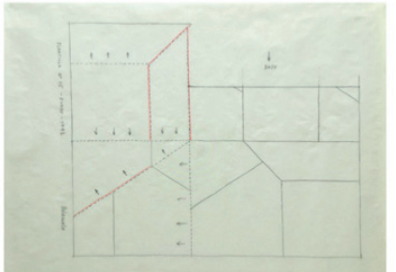

Plantilla No 15 (1992, FPP 46-115)

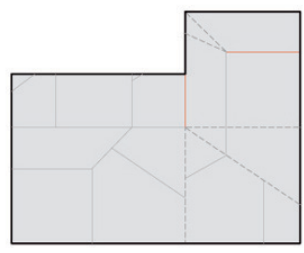

Orto I (1999, FPP 32-029)

Acero con pátina negra $75 \times 60 \times 41 \mathrm{~cm}$

Maqueta $\mathrm{N}^{\circ} 14$

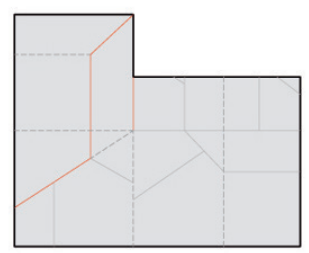

Impulso (1993)

Cobre con pátina negra

$39 \times 60 \times 40 \mathrm{~cm}$

Maqueta $\mathrm{N}^{\circ} 15$

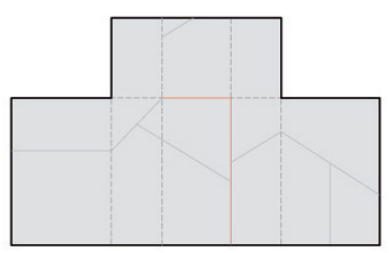

St (c.1990)

Acero cortén

$139 \times 120 \times 75 \mathrm{~cm}$

Maqueta $\mathrm{N}^{\circ} 17$

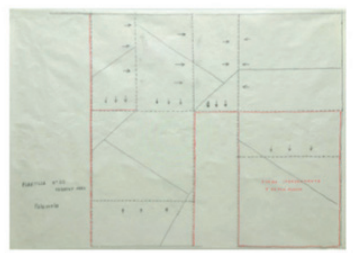

Plantilla $\mathrm{N}^{\circ} 20$

(1992, FPP 46-112)

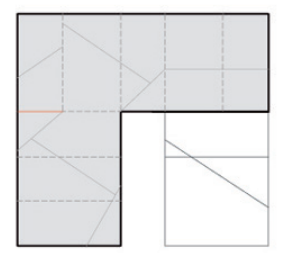

Zigur I (2003)

Acero inoxidable $40 \times 53 \times 36 \mathrm{~cm}$ Maqueta $\mathrm{N}^{\circ} 20$

Fig. 6. Análisis de las trazas de las esculturas de Pablo Palazuelo Impulso (1993), Orto I (1999), Sin título (c. 1990) y Zigur I (2003), realizado por el autor. 


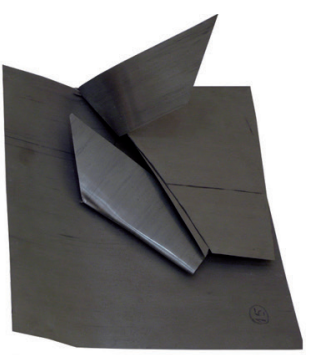

01.

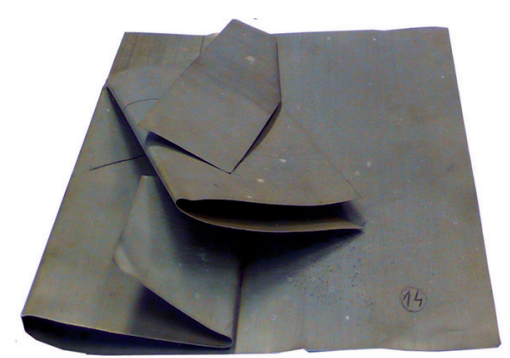

02.

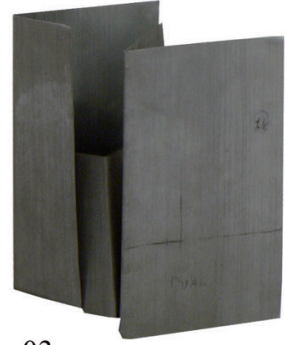

03.

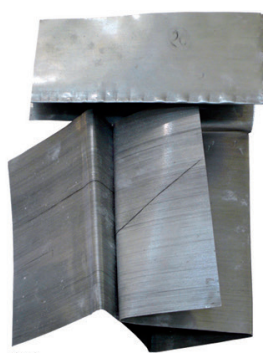

04.

Fig. 7: 01. Palazuelo, Pablo, s.f. Maqueta N. ${ }^{\circ} 15$ [Impulso]. Chapa de zinc. FPP. 02. Palazuelo, Pablo, s.f. Maqueta $N .^{\circ} 14$ [Orto I]. Chapa de zinc. FPP. 03. Palazuelo, Pablo, s.f. Maqueta N. ${ }^{\circ}$ 17. Chapa de zinc. FPP.04. Palazuelo, Pablo, s.f. Maqueta s.t. [Zigur I]. Chapa de zinc. FPP.

y Plantilla $N^{\circ} 14$ (1992, FPP 46-116). Del primero se obtiene una solución que se despliega zigzagueante sobre una base horizontal rectangular. Un proceso recogido tanto en una maqueta de zinc (fig. 7.01), como en la escultura Impulso realizada en 1993.

Idéntico método puede ser sondeado desde la Plantilla $N .^{\circ} 14$ (1992, FPP 46-116) -la cual guarda una relación de simetría con la anterior-, donde se varían la situación de las secciones y el orden de pliegues. De este modo se transformó la coronación de aristas erizadas que presentaba Impulso (1993) en una suerte de librillo plegado sobre un cuadrado horizontal materializado en una primera fase en la maqueta marcada con el número 14 (fig. 7.02). Fruto de este método se elaboró Orto I (1999). Ambas esculturas comparten sendas singularidades: se elevan del suelo sobre un pórtico construido por 3 planchas metálicas y su acabado con pátina negra unifica la variación metálica de cobre y acero que moraba en su interior.

Al variar las secciones eliminadas desde el croquis original por los dos rectángulos simétricos retirados de la Plantilla N. ${ }^{\circ} 17$ (1992, FPP 46-113), se obtuvo una sucesión rectangular semejante a la composición que originó Muro (1998, FPP 32-028). Aunque en esta ocasión todos los polígonos se sitúan en posición vertical para alcanzar una estabilidad próxima a la obtenida por el fuelle de un biombo. Su aspecto sencillo presente en la maqueta de zinc (fig. 7.03) se acentuó durante su salto de escala en acero cortén, en la escultura Sin título (c. 1990).

Como se ha constatado con anterioridad, las transformaciones sufridas por los trazados también implicaban simetrías parciales (fig. 7). De esta manera, la Plantilla $N^{\circ}{ }^{\circ} 19,20$ (1992, FPP 46-109) selecciona una gran porción de dos arquetipos presentes en $N .^{\circ} 14$ (1992, FPP 46-105) y voltea una parte de los otros dos a lo largo de un eje de simetría. A su vez, se manifiesta como contenedor de las trazas para nuevos proyectos.

En el documento titulado Plantilla $N^{\circ} 20$ (1992, FPP 46-112) se produjo un nuevo recorte que secciona el esquema progresivamente hasta convertirlo en una forma de " $L$ " conformada por rectángulos modulados. Con estas directrices, Palazuelo elaboró una maqueta (fig. 7.04) cuya planeidad era desmentida por los pliegues relampagueantes distribuidos en dos direcciones ortogonales del espacio, antesala de la escultura Zigur I (2003).

A partir de un recorte más intensivo del $N .^{\circ} 14$ (1992, FPP 46-105) se desvela un nuevo trazado geométrico que recoge múltiples potencialidades, descrito como ${ }^{\circ}{ }^{\circ} 3,16,21,22,23$ (199293, FPP 46-108). Aunque no se ha encontrado la plantilla específica para el número tres, desde esta fecunda croquización es posible reconstruir el proceso de trabajo que originó la maqueta marcada con dicho número (fig. 8.01). Al operar con un molde de formas, si se destilan las coordenadas ortogonales adecuadas se consiguen los depurados planos verticales que se articulan mediante líneas de corte y pliegue hasta construir la escultura Present I (1997).

Tras sufrir una nueva mutación, se cristalizó el boceto correspondiente a la Plantilla $N .^{\circ} 16$ (1992, FPP 46-114), del que se han hallado conexiones suficientes como para proponerlo como origen de tres piezas escultóricas. Si se mantiene el decidido carácter vertical y el concurso mayoritario de figuras rectangulares, al que se añadió un pliegue diagonal se produjo en 1991 la 


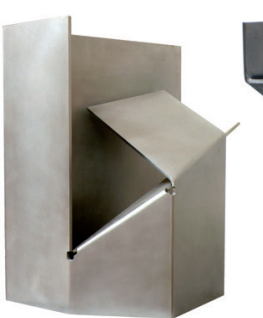

01.

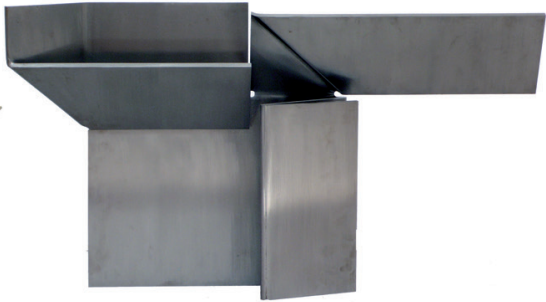

02 .

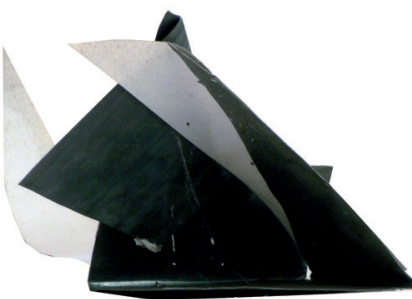

03

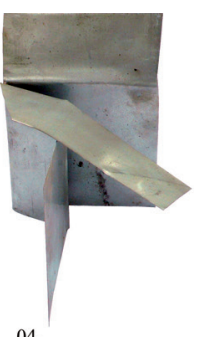

04.

Fig. 9: 01. Palazuelo, Pablo, c. 1991. Envuelto. Acero inoxidable, $65 \times 45 \times 37 \mathrm{~cm}$. FPP 32-016. 02. Palazuelo, Pablo, c. 1991. Planar IV. Acero inoxidable, $32 \times 58 \times 7,5$ cm. FPP 32-013. 03. Palazuelo, Pablo, c. 1998.

Maqueta s.t. [Piramid II]. Chapa de zinc. FPP. 04. Palazuelo, Pablo, s.f. Maqueta N. ${ }^{\circ} 10$. Chapa de zinc. FPP.

pieza Recogimiento (fig. 8.02), donde parecen ensayarse soluciones que se fueron enriqueciendo en las obras posteriores.

La evolución formal prosiguió al tallar los rectángulos siguiendo trazas diagonales que devienen en trapecios, hasta formalizar nuevas subespecies emparentadas con las anteriores (fig. 8). La voluntad vertical previa pervive en las planchas de acero cortén que componen Alada (1993, FPP 32-050), mientras que el proceso de plegado se intensifica para configurar un nuevo prisma abierto. Este triedro se modifica y verifica durante una fase de maqueta, donde se experimentó con diversos materiales para testar las cualidades lumínicas de los reflejos obtenidos, así como los tamaños y posiciones, hasta adoptar la morfología y dimensiones definitivas. Además de la maqueta (fig. 8.03) que se conserva en la Fundación Palazuelo, sus cualidades fueron retomadas para la construcción de Solar II (1996, FPP 32-049).

No es indispensable el seccionado selectivo de los polígonos contenidos dentro de los bocetos para alcanzar ramificaciones esculturales, sino que bastaría con la traducción explícita de los trazos presentes en la Plantilla $N .^{\circ} 14$ (1992, FPP 46-116). Este es el camino seguido en 1994 durante la elaboración de la escultura Shrine I (fig. 8.04), donde se alcanza una máxima correspondencia entre la plantilla y la solución tridimensional, sólo modificada para obtener una inquietante proximidad a la simetría central; propiedad rechazada sistemáticamente dentro de la obra de Palazuelo. Un triedro se pliega asistido por múltiples aristas de sección de las que destacan los agudos triángulos ensimismados que apuntan al corazón de la composición.

Dentro del usual método de clarificación de los trazados reguladores, Palazuelo separó las pautas contenidas en el diseño anterior (FPP 46-117) hasta destilar algunos de los moldes formales que moraban entre sus líneas. Aislados a partir de la medianera horizontal, la porción inferior conformada por un arquetipo y parte del adyacente configuraban la Plantilla $N^{\circ} 9$ (1991, FPP 46-120), donde habitaban sendas esculturas. Como un pentágono abierto, los planos verticales limitan el espacio plegado en torno a la pieza Envuelto (fig. 9.01), donde la simetría se rompe gracias a un pliegue diagonal en una de sus superficies.

Por otro lado, la mitad superior del boceto primigenio desembocó en la Plantilla N. ${ }^{\circ} 8$ (1991, FPP 46-119), cuyo trazado regulador fue empleado para la producción de Planar IV (fig. 9.02). Una escultura de modestas dimensiones que abundaba en la investigación de las posibilidades que habilitaban los pliegues sobre el plano vertical, pero que contraponía el despliegue de una coronación con un marcado carácter horizontal.

La pregnancia de estos diseños acompañó la obra del escultor madrileño a lo largo de dilatados períodos. De este modo, la anterior plantilla (FPP 46-119) fechada en 1991 prolongó sus diagonales hasta reconstruir triángulos ortogonales que concursaron en la elaboración de una maqueta, que conserva las huellas de su método de producción. Sobre ella aún permanecen pegados los recortes de papel sobre la superficie de zinc (fig. 9.03), que obraron como plantilla de corte para facilitar su modelado. Un sistema de trabajo que Palazuelo enumeró a Obrist: "A veces utilizo fragmentos de dibujos que hice inicialmente para un cuadro y los corto a ciegas. Los re- 


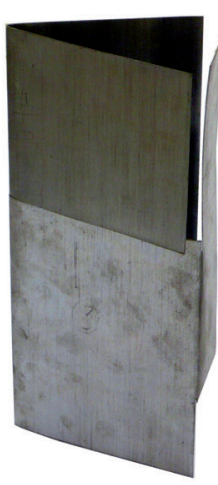

01.

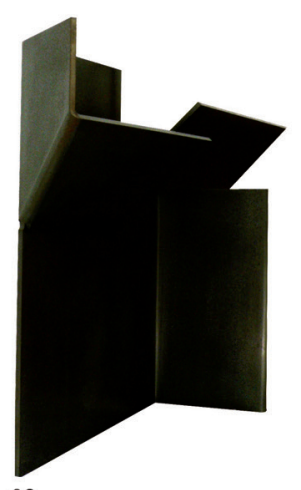

02.

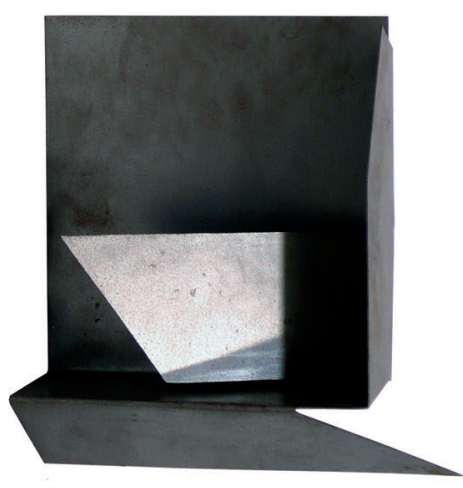

03.

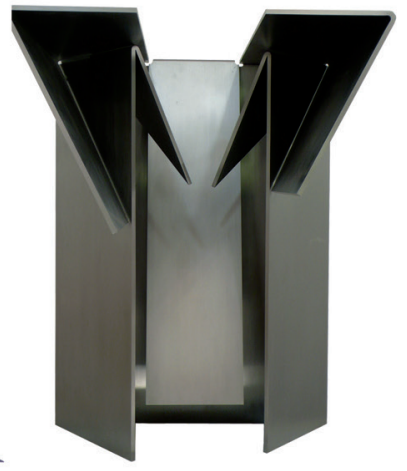

04.

Fig. 8: 01. Palazuelo, Pablo, s.f. Maqueta N. 3 [Present I]. Chapa de zinc. FPP. 02. Palazuelo, Pablo, 1991. Recogimiento. Acero cortén pulido y fregado, $120 \times 210 \times 51 \mathrm{~cm}$. FPP 32-047. 03. Palazuelo, Pablo, s.f. Maqueta s.t. [Solar II]. Chapa de zinc. FPP. 04. Palazuelo, Pablo, 1994. Shrine I. Acero inoxidable, $80 \times 60 \times 74 \mathrm{~cm}$. FPP 32-017.

corto y los pego sobre un papel normal. Entonces trabajo con una hoja de aluminio muy fina -casi tan fina como una hoja de papel-, lo que me permite recortarla con facilidad" ${ }^{36}$.

En la escultura resultante, Piramid II (2000), sus polígonos de marcado componente diagonal gravitan alrededor de una base rectangular para obtener un fulgurante juego de reflejos gracias al brillante acabado metálico del bronce.

Entre las plantillas que habitaban en el trazado de $N .^{\circ} 1,5,7,10,11,12$ (1991, FPP 46-104), el presente estudio fija su atención sobre las dos últimas que han sido objeto de análisis, concretamente las que ostentan los números 10 y 12. De estos trazados sólo se ha constatado la existencia de una escultura, aunque existen importantes vestigios del proceso de trabajo empleado que sirven para descifrar nuevos mecanismos de proyecto.

En la Plantilla $N^{\circ} 10$ (1991, FPP 46-121) se puede apreciar una evolución respecto a la plantilla número ocho antes comentada, al variar la dirección diagonal de corte, además de apostar por la seriación rectangular. De esta propuesta se han hallado tanto un modelo tridimensional (fig. 9.04), donde se configura un diedro vertical que soporta una coronación trapezoidal, como las notas manuscritas por Casanovas. En estos apuntes se especificaba la intención de operar con planchas de sección variable, donde el grosor disminuía hacia los extremos, conservando el mayor índice de masa en un núcleo que reforzaba la composición centrípeta.

\section{Conclusiones}

Con el recorrido trazado en este artículo se ha tratado de desvelar algunos de los aspectos principales operados durante el método productivo de Palazuelo al enfrentarse a las tres dimensiones. El motivo para la elección de estas realizaciones obedece a un doble planteamiento: poder contrastar la documentación registrada durante la investigación, estableciendo conexiones entre sus obras; y, por otra parte, seleccionar las piezas más representativas que ilustrasen las diversas genealogías desarrolladas a partir de los trazados Del cuatro, donde habitaba el mayor número de posibilidades formales desarrolladas durante su aventura escultural.

En anteriores publicaciones acerca de la escultura de Palazuelo el foco se ha centrado principalmente en la obra conclusiva y terminada. Este ensayo no sólo presenta material inédito, sino

\footnotetext{
36 Pablo Palazuelo, citado en Obrist, 2005: 41-42.
} 
que también permite por primera vez la reconstrucción relacional de las huellas gráficas y maquetas que ilustran el proceso implementado. Se incide en un período de madurez en el que Palazuelo presentaba una concepción espacial complementaria a la escultura volumétrica de Chillida y a las superficies vaciadas de Oteiza. En sus esculturas se determinaba una envolvente como resultado del lenguaje procesal de pliegues, donde más que definir el espacio, lo tensionaba operando sobre los paramentos que lo confinan.

El concurso de una red estructuradora de base rectangular complementada con la inclusión de diagonales plantea una variedad de combinaciones prácticamente inagotable. Un método que difiere sustancialmente de mallas cuadradas empleadas por Oteiza para generación de las unidades Málevich, las cuales delimitaban los contornos de sustracción de material. Dado que el orden previo impuesto en la geometría mística elegida por Palazuelo determina la formalización de las aristas de corte y de pliegue, su producción parece representar una aproximación plástica a las evoluciones de los sistemas caóticos estudiados por la física.

A través de esta muestra se ha podido vislumbrar el lenguaje polisémico presente en la croquización de Palazuelo, donde las combinaciones de dos arquetipos permiten un amplio espectro de soluciones espaciales con propiedades equipotenciales. Sin embargo, este estudio no ha sido completamente agotado, por el contrario se ha pretendido proporcionar las herramientas necesarias que permitan desvelar nuevos hallazgos en futuras líneas de investigación, a partir de las exploradas por Palazuelo a lo largo de su fecunda producción.

\section{BIBLIOGRAFÍA}

Arangüena, Alfonso (ed.) (1987): Palazuelo. Madrid: Galería Theo.

Barañano, Kosme (ed.) (1998): Chillida 1948-1998. Madrid: Museo Nacional Centro de Arte Reina Sofía.

Bohm, David (1980): Wholeness and the Implicate Order. Londres: Routledge \& Kegan Paul.

Bouleau, Charles [1963] (1988): La geometria segreta dei pittori. Milán: Electa.

Chipp, Herschel B. [1968] (1995): Teorías del arte contemporáneo. Fuentes artísticas y opiniones críticas. Madrid: Akal. Deleuze, Gilles [1988] (1989): El pliegue. Leibniz y el Barroco. Barcelona: Paidós.

Franz, Marie Louise von [1970] (1974): Number and Time. Evanston: Northwestern University Press.

Gállego, Julián (1976): "Los nombres de Palazuelo”. En: Revista de Occidente, Tercera época, número 7, Madrid, pp. 5358.

Ghyka, Matila [1946] (1977): The Geometry of Art and Life. Nueva York: Dover.

Guigon, Emmanuel/Llorens, Tomás (eds.) (1998): Paul Klee. Valencia: Instituto Valenciano de Arte Moderno, Museo Thyssen Bornemiza.

Ivanova-Veen, L. I./Ovsiannikova, E. B. (2005): From VKhuTEMAS to MARKhl. 1920-1936. Moscú: A-Fond Publishers. Khanna, Madhu/Mookerjee, Ajit (1979): Yantra: The tantric symbol of cosmic unity. London: Thames and Hudson.

Le Corbusier [1923] (1946): Towards a New Architecture. Londres: Architecture Press.

Obrist, Hans (2005): "Palazuelo de la A a la Z. (Pars pro toto)". En: Pablo Palazuelo 1995-2005. Madrid: Museo Nacional Centro de Arte Reina Sofía, pp. 40-43.

Palazuelo, Pablo (1985): Palazuelo. Pinturas, esculturas, gouaches. Madrid: Galería Theo.

Palazuelo, Pablo (1999): Pablo Palazuelo. Esculturas, dibujos y gouaches 1993-1998. Madrid: Fábrica Nacional de Moneda y Timbre.

Palazuelo, Pablo/Entrevistado por Amón, Santiago (1976): "Palazuelo: materia, forma y lenguaje universal”. En: Revista de Occidente, Tercera época, número 7, Madrid, pp. 24-35.

Palazuelo, Pablo/Esteban, Claude (1980): Palazuelo. Barcelona: Maeght.

Palazuelo, Pablo/Entrevistado por Guisasola, Félix (1981): "Conversación con Palazuelo". En: Q Revista del CSCAE, número 44, Madrid, pp. 19-26.

Palazuelo, Pablo/Power, Kevin (1995): Geometría y Visión. Una conversación con Kevin Power. Granada: Diputación Provincial de Granada.

Penrose, Roger [1989] (1991): La nueva mente del emperador. Madrid: Mondadori.

Rowell, Margit/Badiola, Txomin (2005): Oteiza: mito y modernidad. Madrid: FMGB, SEACEX y Fundación Museo Guggenheim Bilbao.

Sotelo, Gonzalo/Oropesa, Marisa (2012): Pablo Palazuelo inédito. La colección del artista. Valladolid: Ayuntamiento de Valladolid.

Stewart, Ian [1989] (1991): ¿Juega Dios a los dados? La nueva matemática del caos. Barcelona: Crítica.

Fecha de recepción: 26-I-2015

Fecha de aceptación: 22-VI-2015 